"This is an Accepted Manuscript of an article published by Polymers for Advanced Technologies (PAT), of Wiley, vol 31 (issue no 7), pgs 1474-1483, accepted on September 19, 2019."

\title{
Evaluation of Doped and Undoped Poly (o-anisidine) as Sensing Materials for a Sensor Array for Volatile Organic Compounds
}

\author{
Katherine M. E. Stewart ${ }^{1,2 *}$, Alison J. Scott ${ }^{1}$ and Alexander Penlidis ${ }^{1}$ \\ 1 Institute for Polymer Research, Department of Chemical Engineering, University of Waterloo, Waterloo, Ontario, \\ Canada, N2L 3G1 \\ 2 Currently with the Department of Chemistry and Physics, Troy University, Troy, Alabama, USA, 36082 \\ * Correspondence: kastewart@troy.edu
}

\begin{abstract}
Poly (o-anisidine) (PoANI) and PoANI doped with nickel oxide and zinc oxide were evaluated as sensing materials for four gas analytes (methanol, ethanol, acetone, and benzene). The sensing materials had high sensitivity (showing an affinity towards the target analytes even at low concentrations, in the range of $1 \mathrm{ppm}$ to $5 \mathrm{ppm}$ ), but rather poor selectivity, especially when the gas analytes were in a mixture. To exploit the poor selectivity, the three sensing materials were combined into a sensor array using principal component analysis (PCA) as a sensing algorithm. It was found that using a sensor array, the four individual gases could be separated. However, when all four gases were present (in analyte mixtures), there was too much overlap in the responses to distinguish between individual gas analytes and their related mixtures.
\end{abstract}

Keywords: Poly (o-anisidine); Nickel oxide (NiO); Zinc oxide (ZnO); Gas sensor array; Principal Component Analysis (PCA)

\section{Introduction}

Sensor arrays or electronic noses (e-noses) have been developed for a wide variety of applications, including discrimination between and detection of specific ingredients in food ${ }^{1,2}$ and beverage ${ }^{3,4}$ industries, wastewater assessment ${ }^{5}$, disease diagnosis ${ }^{6,7}$, and chemical process analysis ${ }^{8}$. Sensor arrays combine multiple sensing materials into one sensor. The response from each of the sensing materials is incorporated into some algorithm (often an artificial neural network), which analyzes all of the data and provides an output where specific analytes are identified. ${ }^{5}$ For an effective sensor array, both the algorithm and the sensing materials are important.

The algorithms are used in principle to discriminate between different analytes through pattern recognition. Fine-tuning an algorithm can improve the selectivity of a sensor array for a specific application. ${ }^{9}$ However, as a proof of concept, widely available cluster analysis tools, such as principal component analysis (PCA), are used by researchers. ${ }^{10,11}$ 
When selecting sensing materials, the specific materials do not need to exhibit high selectivity towards different analytes (that is, they do not need to sorb one specific analyte significantly more than other competing or interfering analytes). However, the response pattern should be different from that of the other sensing materials used. ${ }^{12}$ A pattern with a larger variation in sensing material response to gas analytes generally means that fewer sensing materials may be needed in a sensor array. ${ }^{13}$ In other words, if two materials respond similarly to gas analytes (sorbing approximately equal amounts of a given analyte, showing similar affinity to specific gas mixtures, etc.), incorporating both materials into a sensor array may not show significant improvement in terms of gas analyte separation and/or identification (compared to only using one of the materials in an array). Rather, in developing a sensor array, the goal is to find materials that respond uniquely; if material A preferentially sorbs a different analyte than material B, using the two materials in a sensor array will complement each other and enrich the information content of the array. Therefore, synthesizing, characterizing and evaluating sensing materials for various applications and environments are all important steps/aspects for the development of new sensor arrays.

Poly (o-anisidine) (PoANI) is a derivative of polyaniline and has been used as a sensing material for a variety of volatile organic compounds (VOCs), including aliphatic alcohols and aldehydes. ${ }^{14-}$

${ }^{16}$ Since it has been used as a sensing material for multiple VOCs, it has partial selectivity. This means that PoANI can respond somewhat non-discriminately to a range of gases; this non-specific sorption can be advantageous for sensor arrays, since it shows a partial response to several different gas analytes. However, to provide some specificity (that is, to improve the selectivity of PoANI), the material was often doped with nickel (II) oxide (NiO) and zinc oxide ( $\mathrm{ZnO}) .{ }^{10,17}$ Selectivity is a measure of how much a sensing material favours a target analyte over other possible interferents (or competing gases) and can be calculated as per Equation 1.

$$
\text { Selectivity }=\frac{\text { Target } \text { Analyt }_{\text {sorbed }}}{\text { Interferent }}
$$

These three polymeric materials (pure PoANI, PoANI doped with NiO, and PoANI doped with $\mathrm{ZnO}$ ) were evaluated as sensing materials for methanol, ethanol, acetone, and benzene. In addition, a sensor array was assessed using these three sensing materials and PCA as the 'clustering' algorithm.

\section{Experimental}

\subsection{Material Synthesis}

The poly (o-anisidine) (PoANI) nanocomposites were synthesized by mixing o-anisidine (monomer), ammonium persulfate (initiator), and (if present), the dopants in deionized water. All chemicals were used as received. Up to $0.41 \mathrm{~mL}$ of $o$-anisidine (A.C.S. reagent, Sigma-Aldrich, Oakville, Ontario, Canada) was added to $20 \mathrm{~mL}$ of deionized water along with the metal oxide dopant (20 wt. \% of the total polymeric sample weight). Two dopants were used: nickel (II) oxide (particle size $<50$ nm, 99.8 \% purity, Sigma-Aldrich, Oakville, Ontario, Canada) and zinc oxide 
(ZnO) (particle size <100 nm, 50 wt. \% in water, Sigma-Aldrich, Oakville, Ontario, Canada). The (doped) monomer solution was mixed using a sonicator for 30 minutes. It was then cooled to $-1^{\circ} \mathrm{C}$ before adding a solution containing $1.0 \mathrm{~g}$ of ammonium persulfate (A.C.S. Reagent, SigmaAldrich, Oakville, Ontario, Canada) in $5 \mathrm{~mL}$ of deionized water. The resulting solution was shaken by hand for 1 minute to ensure thorough mixing, then left to polymerize (at $-1^{\circ} \mathrm{C}$ ) for 6 hours. The polymer was filtered (out of the solution) using a Büchner funnel and Whatman \#5 filter paper, washed with acetone, and subsequently left overnight to dry in air. The PoANI nanocomposites were stored under atmospheric conditions in $20 \mathrm{~mL}$ scintillation vials. ${ }^{18}$

\subsection{Gas Sorption Test System}

A specially designed gas test system was used to evaluate the sorption capabilities of different potential sensing materials. The set-up exploits a difference in gas concentration (before and after exposure to the sensing material) to establish how much of the target analyte has been sorbed. The test system consists of an analyte source (standard grade mixtures of a gas analyte in a balance of nitrogen) in gas cylinders, with the gas flow controlled by MKS RS-485 mass flow controllers (MFCs). The gas flows through an MKS 640A pressure controller (PC) and an MKS 1179A flow meter (FM) to ensure the pressure remains above 15 psi and that the flow rate is maintained at 200 sccm. Each gas analyte (or gas analyte mixture) passes through a $100 \mathrm{~mL}$ round bottom flask, which contains a sample of the sensing material.

Before the gas analyte flows over a specific sensing material, a 'blank' run can be performed (an empty flask is used to establish the baseline). Results are collected using a highly accurate Varian 450 gas chromatograph (GC) (with a specialized photon discharge helium ionization detector (PDHID)), which determines the gas concentration for the case of no sorption. When the sensing material is present, i.e., once the empty flask is replaced with a flask containing polymeric material, the gas stream again flows into the GC, which can distinguish between similar analytes and record concentrations down to the parts-per-billion (ppb) level (see Figure 1). ${ }^{19}$

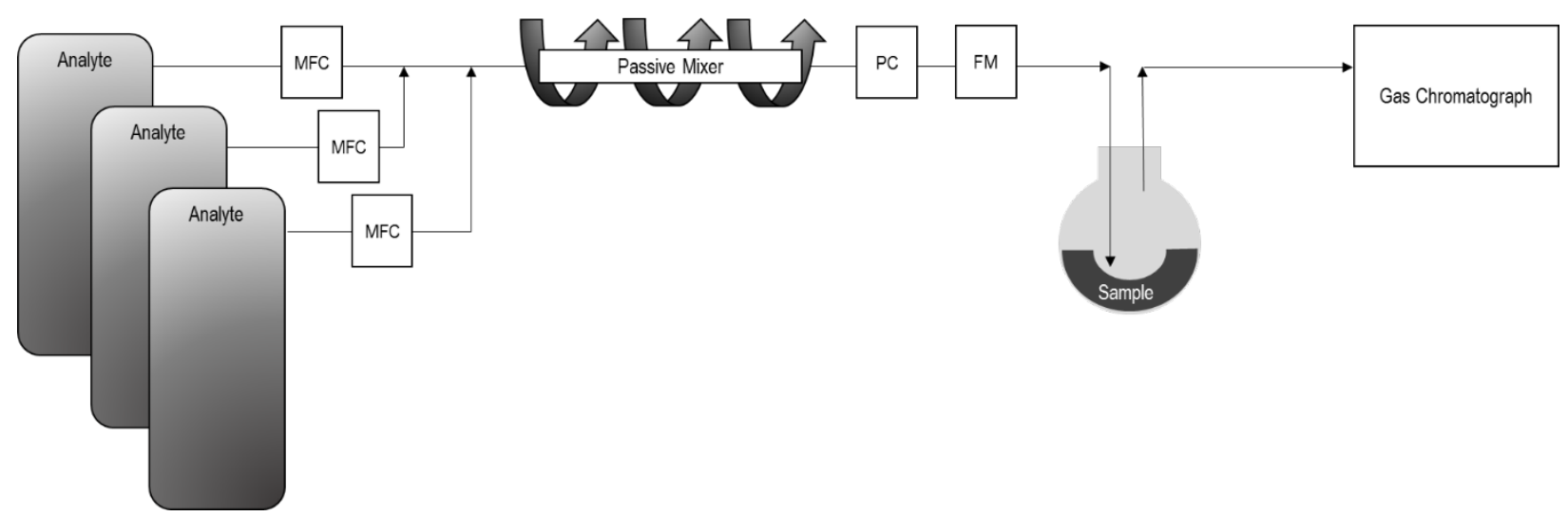

Figure 1 Experimental test set-up, where MFC, PC, and FM are mass flow controller, pressure controller, and flow meter, respectively. 


\subsection{Evaluation of Potential Sensing Materials}

Test samples of each PoANI nanocomposite were prepared by adding $0.120 \mathrm{~g}$ of sample to a 100 $\mathrm{mL}$ round bottom flask with $5 \mathrm{~mL}$ of ethanol. Each sample was swirled around the flask to coat the interior of the flask, and then placed in an oven at $50^{\circ} \mathrm{C}$ for 18 hours. The samples were cooled to room temperature $\left(21^{\circ} \mathrm{C}\right)$ before being tested.

Prior to evaluation, each sample was purged with dry nitrogen (5.0 grade, Praxair, Mississauga, Ontario, Canada) for 60 minutes. This purge was conducted immediately before a sample was exposed to an analyte. Four gas analytes were used to evaluate the effectiveness of these polymeric nanocomposites as sensing materials. These gas analytes were all approximately $5 \mathrm{ppm}$, standard grade mixtures in a balance of nitrogen (Praxair, California, USA): methanol (4.66 ppm), ethanol (5.00 ppm), acetone (5.50 ppm) and benzene (5.10 ppm).

The PoANI nanocomposites were evaluated (at room temperature, around $21^{\circ} \mathrm{C}$ ) by exposing each PoANI nanocomposite to specific concentrations of different gas analytes (methanol (M), ethanol (E), acetone (A), and benzene (B)) individually and in mixtures (up to all four gases). Mixtures containing approximately 1 ppm (three- and four-gas mixtures), 2 ppm (two-gas mixtures), and 5 ppm (individual gases) of each analyte were used and the PoANI nanocomposites were exposed to the gas for at least 60 minutes to ensure that equilibrium had been reached. The amount that did not sorb onto the PoANI nanocomposite was measured using the highly specialized GC. By subtracting this amount from the initial concentration exposed (from the 'blank' run verification measurement), the amount of gas analyte that sorbed onto each PoANI nanocomposite was ascertained. Three independent replicates were conducted for each polymeric nanocomposite for each gas tested.

\section{Results and Discussion}

\subsection{Sorption Performance}

The three sensing materials (PoANI, PoANI 20\% NiO, and PoANI 20\% ZnO) were independently exposed to each of the four (individual) gas analytes (methanol, ethanol, acetone, and benzene); sorption results are shown in Figure 2. In terms of sensitivity, PoANI 20\% ZnO showed the highest sorption, which indicates that it was the most sensitive (had the highest affinity to most of the gases). In comparing the sorption of the four gas analytes, PoANI 20\% ZnO was most sensitive to methanol (that is, methanol had higher sorption onto PoANI 20\% ZnO compared to the other analytes). However, the selectivity of the PoANI 20\% ZnO should also be considered. Since methanol had the highest sorption for PoANI 20\% ZnO, we can designate it the target analyte (as per Equation 1). The selectivity towards methanol was 1.1 with respect to ethanol $\left(\mathrm{M}_{\text {sorbed }} / \mathrm{E}_{\text {sorbed }}\right.$ $=1.1$ ), 1.5 with respect to acetone, and 7.1 with respect to benzene. This indicates that the selectivity of PoANI 20\% ZnO towards methanol was low with respect to ethanol and acetone, but high with respect to benzene. Therefore, despite good sensitivity, PoANI 20\% ZnO exhibited poor selectivity. 
In contrast, PoANI had the lowest sensitivity (lowest affinity) for all four gas analytes (this is evidenced by the significantly lower amount of each analyte sorbed compared to both NiO-doped and ZnO-doped PoANI. This validates the decision to add metal oxide dopants to the pure PoANI, since both metal oxide-doped materials increase the amount of each analytes sorbed. As shown in Figure 2, PoANI promoted acetone sorption more than the sorption of other gas analytes (therefore, it was partially selective towards acetone). Specifically, PoANI had a selectivity towards acetone of 2.5 with respect to methanol, 2.0 with respect to ethanol, and 8.8 with respect to benzene (note that these calculations consider acetone as the target analyte, since it is sorbed in the largest proportion, and that $\mathrm{A}_{\text {sorbed }}$ would be used in the numerator as per Equation 1). Given this information, PoANI can be useful in a sensor array (which will be discussed in what follows), but the low sorption (poor sensitivity) and low selectivity suggest that PoANI would be a poor standalone sensing material for these analytes. For many applications, selectivity of 2.0 is not high enough, as it may result in too many false positives.

Finally, PoANI 20\% NiO had moderate sensitivity (better than PoANI, but generally lower sorption than PoANI 20\% ZnO), and selectivity was poor (all four gas analytes showed similar sorption). As for the PoANI 20\% ZnO, methanol had the highest sorption. Interestingly, even though most analytes demonstrated lower sorption on the NiO-doped material (compared to the $\mathrm{ZnO}$-doped material), the benzene sorption was twice as high for the $\mathrm{NiO}$-doped material (again, compared to the $\mathrm{ZnO}$-doped material). This difference in material responses should enrich the data available for multivariate analysis (that is, the development of a sensor array).

Overall, all three sensing materials were rather poor with respect to selectivity on their own, but provide a wealth of information that can be combined in a sensor array.

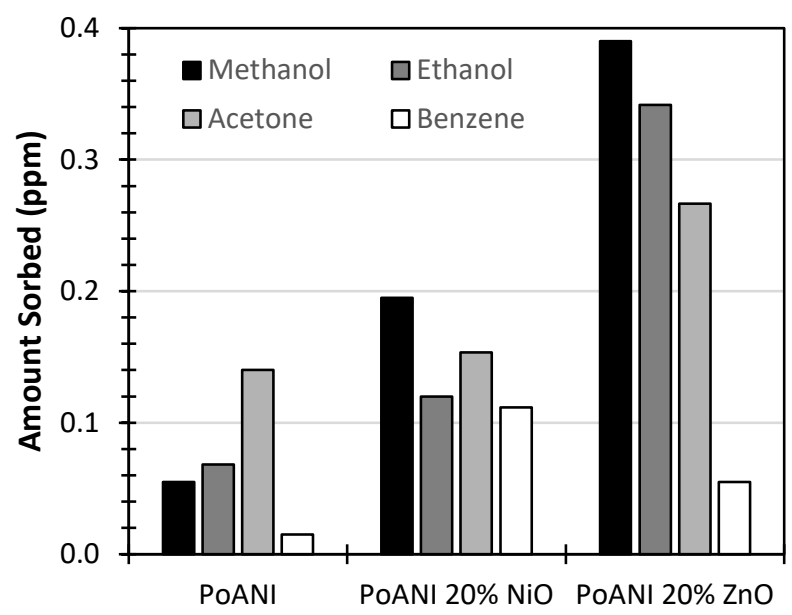

Figure 2 The amount of each gas analyte (methanol, ethanol, acetone, and benzene) sorbed onto each sensing material (PoANI, PoANI 20\% NiO, and PoANI 20\% ZnO) when each analyte was tested individually. 
Since multiple analytes are often present in the environment where chemical sensors are used, the sensing materials were exposed to different combinations of the gas analytes simultaneously. Figure 3 shows the material responses when two-analyte combinations were used, Figure 4 shows material responses for three-analyte combinations, and Figure 5 gives each material's response to the mixture of all four gas analytes (exposed to a given polymeric sensing material simultaneously).
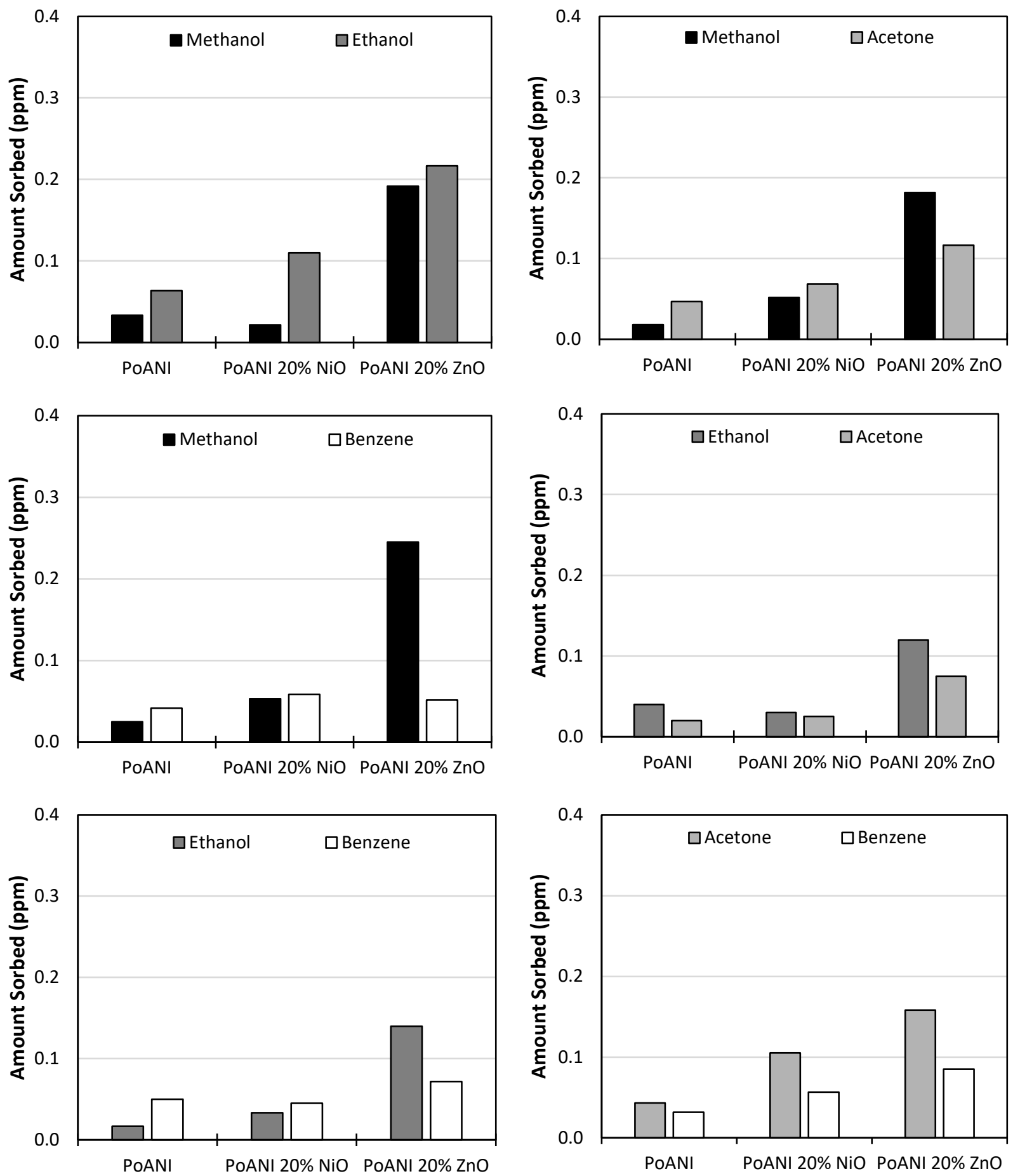
Figure 3 The amount of each gas analyte (methanol, ethanol, acetone, and benzene) sorbed onto each sensing material (PoANI, PoANI 20\% NiO, and PoANI 20\% ZnO) when two analytes were present in the gas mixture.
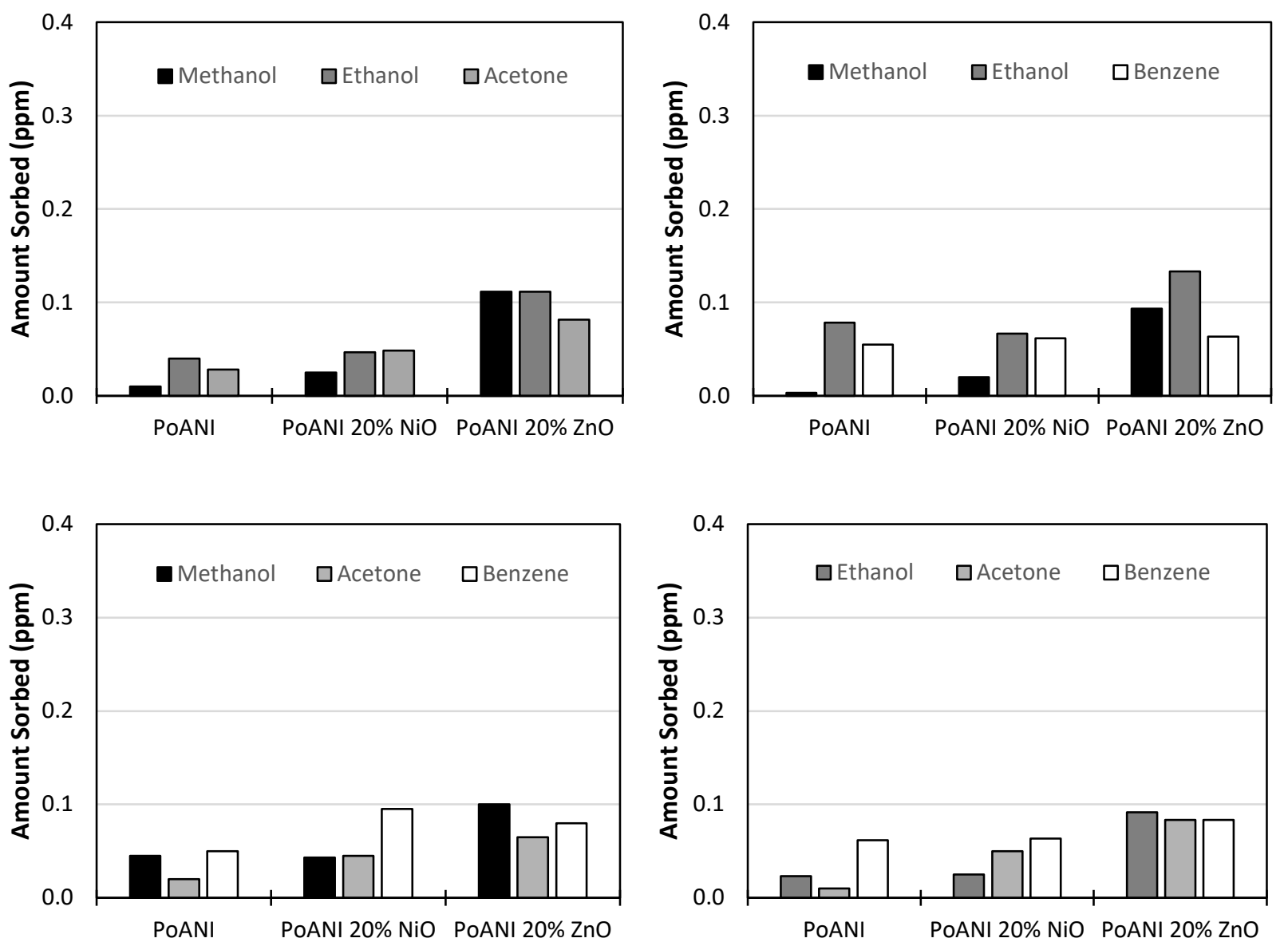

Figure 4 The amount of each gas analyte (methanol, ethanol, acetone, and benzene) sorbed onto each sensing material (PoANI, PoANI 20\% NiO, and PoANI 20\% ZnO) when three analytes were present in the gas mixture. 


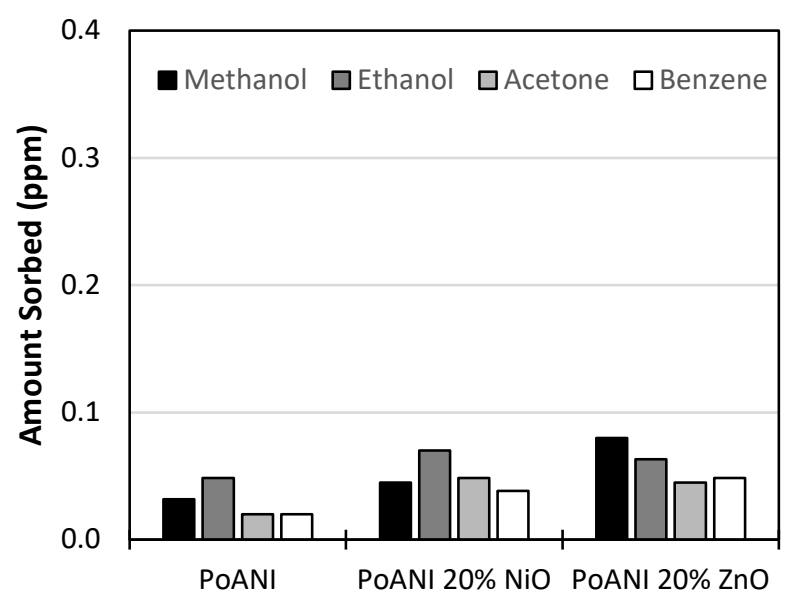

Figure 5 The amount of each gas analyte (methanol, ethanol, acetone, and benzene) sorbed onto each sensing material (PoANI, PoANI 20\% NiO, and PoANI 20\% ZnO) when all four analytes were present in the gas mixture.

When gas mixtures are used, the concentration of each analyte in the 'source' gas necessarily decreases (recall that the individual gases contain $\sim 5 \mathrm{ppm}$ of the target analyte, but that three- and four-gas mixtures only contain $\sim 1 \mathrm{ppm}$ of each analyte present in the mixture). This immediately reduces the amount of analyte molecules that are available to sorb. Also, as more gas analytes are simultaneously exposed to a sensing material, they compete for available sorption (sensing) sites. ${ }^{20}$ This often results in lower sorption of each gas analyte, which can be seen in Figures 3 through 5.

As shown in the progression of Figures 2 through 5, the sorption of each gas analyte often decreases as the number of gas analytes increases. For example, PoANI 20\% ZnO had an ethanol sorption of almost $0.4 \mathrm{ppm}$ when ethanol was the only analyte (Figure 2), but the response to ethanol dropped to an average of $0.2 \mathrm{ppm}$ when two analytes were present simultaneously (Figure 3 ), then down to an average of $0.1 \mathrm{ppm}$ when three-gas mixtures were tested (Figure 4), and finally down to $0.08 \mathrm{ppm}$ when all four analytes were simultaneously present in the gas mixture (Figure 5).

The reduced affinity toward each gas analyte as more analytes are present in the mixture often results in lower selectivity (values much closer to 1.0). The poorer selectivity can be seen in Figures 3 through 5, compared to the responses of individual gases (Figure 2). Poor selectivity, especially when a material is exposed to multiple gas analytes simultaneously (as in a real-world application), means that each sensing material cannot be used individually for a sensor. However, these sensing materials do sorb these analytes at room temperature and thus, could be used in a different application either as a sensor array (to be discussed shortly) or as an absorbent to remove analytes from air. 


\subsection{Sensor Array}

One way to improve the performance of a sensor is to create a sensor array. A sensor array exploits low selectivity of sensing materials by combining the response of multiple materials. The goal is to create a signature for an analyte or mixture using a data processing algorithm. ${ }^{21}$

As a proof of concept, principal component analysis (PCA) was used as the data processing algorithm to separate the gas analyte signatures from these three sensing materials. PCA is a wellknown multivariate tool, often used to determine the inter-relationships between variables in large data sets. The goal is to explain the variation of a data set with fewer factors than were originally provided. By reducing the number of factors (components) in the data set, PCA simplifies the analysis while retaining the majority of the statistical information. This is useful for providing an understanding of relationships within and between variables and is often useful for troubleshooting and/or process analysis. ${ }^{22}$ For example, plotting data often reveals clusters of data, indicating a collection of experimental runs/trials had similar properties and, in some cases, outlying trials. In addition, PCA can be used to identify influential variables and provide a qualitative idea of the relationships within or between variables.

PCA was applied to the responses from the individual gas analytes (that is, the sorption measurements from Figure 2) and two principal components (or factors) were plotted against each other (see Figure 6). As a reminder, in Figure 6 (and throughout this work), M stands for methanol, E stands for ethanol, A stands for acetone, and B stands for benzene. It should be noted that for the individual gases, Factor 1 vs. Factor 3 was used, whereas Factor 1 vs. Factor 2 was used for all of the other PCA plots. In general, Factor 1 vs. Factor 2 provides the best separation (since the first two factors contain the most statistical information). However, when using PCA as an algorithm for sensor arrays, these two factors may not always provide the best separation of analyte signatures. It is more important for a sensor array to have good separation of analytes than it is to maximize information content with fewer factors. This motivated the decision to plot Factor 1 versus Factor 3 for the individual gases (Figure 6) and Factor 1 versus Factor 2 for the gas mixture analyses (Figures 7-11). 


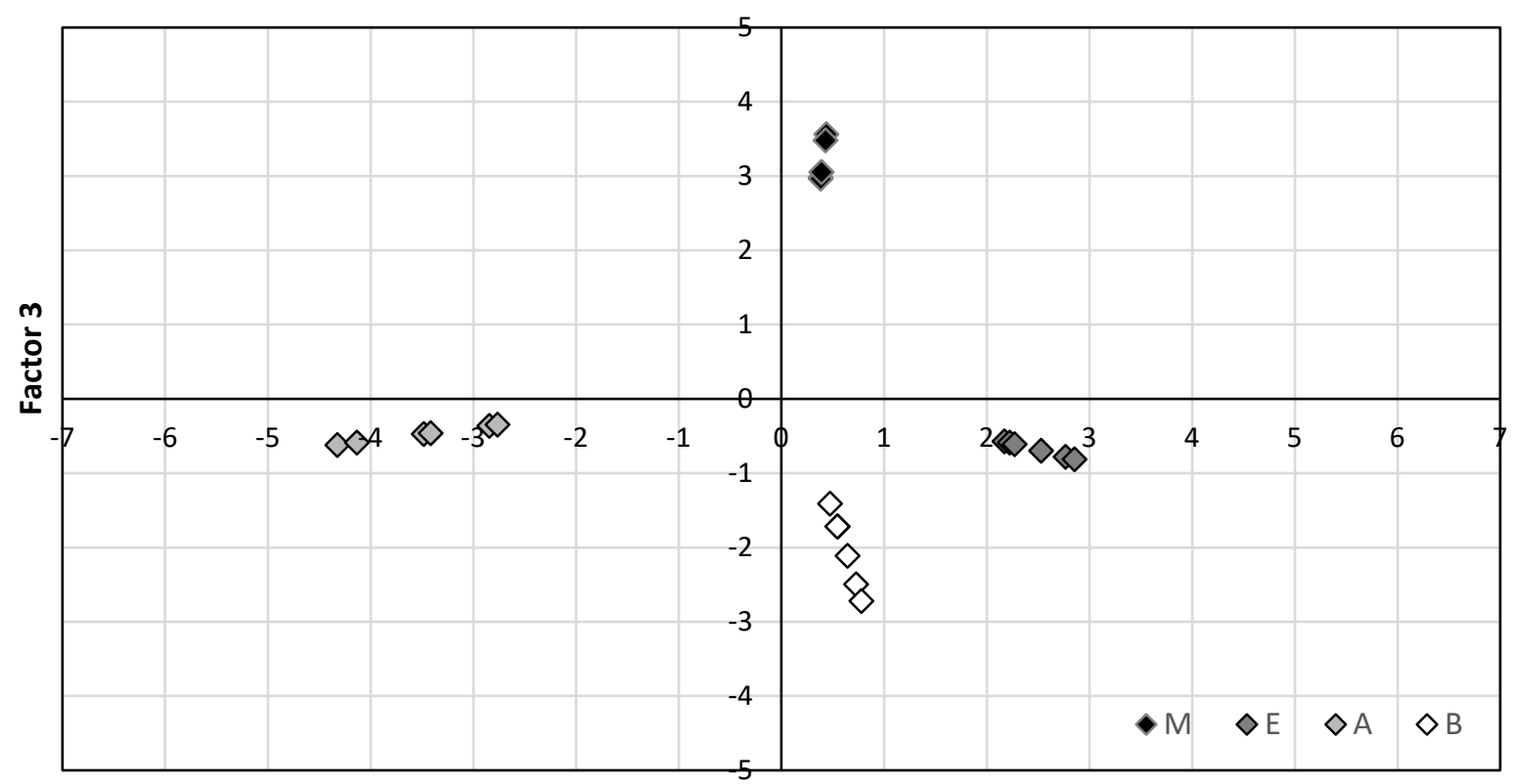

Factor 1

Figure 6 Sensor array results for individual gases of methanol (M), ethanol (E), acetone (A), and benzene (B).

For individual gas analytes (as in Figure 2), these three PoANI-based sensing materials (along with PCA as a clustering algorithm) are able to differentiate between methanol, ethanol, acetone and benzene. However, for real world applications, gas analytes are always present with other gas analytes (interferents). Therefore, the various possible mixtures of these four gas analytes must be considered. PCA was applied to all of the related combinations of gas analytes with the goal of using these sensing materials and PCA in a more realistic setting (see Figure 7). 


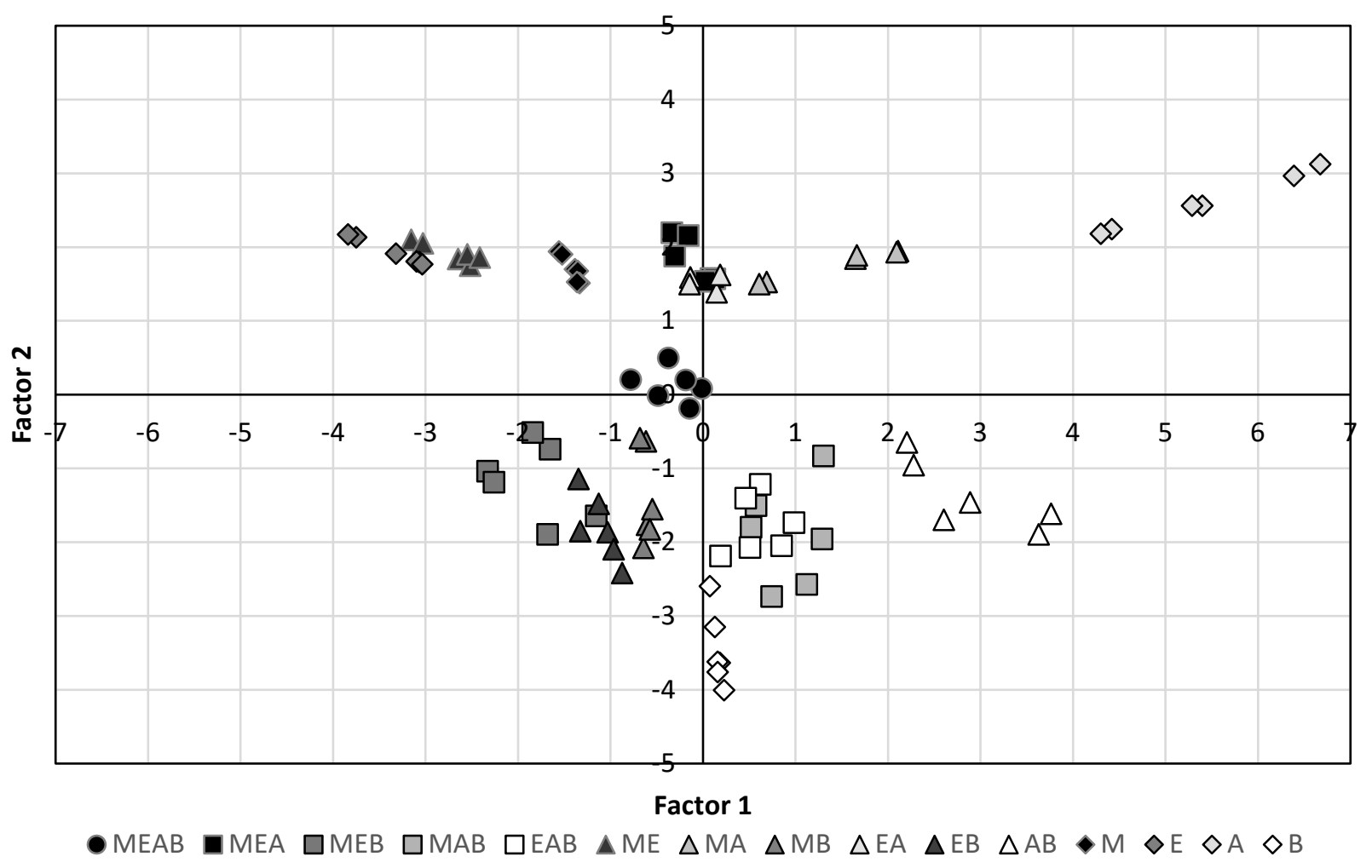

Figure 7 Sensor array results for all possible gas mixture combinations of methanol (M), ethanol (E), acetone (A), and benzene (B).

From Figure 7, we can see the clustered responses of all four (individual) gas analytes and all of the gas mixtures (composed of two, three or four of the analytes). The individual gas responses (shown as diamonds in Figure 7) are generally on the outskirts of the plot; methanol and ethanol are in the top left quadrant, acetone is in the top right quadrant, and benzene is central in the lower half of the plot. As discussed earlier, the individual gases typically have the highest amount of sorption (the sorption of each gas analyte decreases as the number of gas analytes in the mixture increases). The larger response of the pure gases is somewhat amplified by the PCA projection, which explains why the clusters related to individual gases are on the periphery in Figure 7.

Upon closer inspection, Figure 7 also shows the relationship between certain gas analytes and gas mixtures. For example, it is not coincidental that the four-gas mixture of methanol, ethanol, acetone, and benzene (MEAB, circles in Figure 7) is in the centre of the plot. The response to the MEAB mixture is centrally located between each of the individual analytes because it contains attributes of each of the four gases. Similarly, if we look at a simpler case (like a two-gas mixture of methanol and ethanol), the ME response falls in between the pure methanol and the pure ethanol responses. In plotting these factors (Factor 1 and Factor 2 from PCA), methanol and ethanol give similar responses. Because they are close together, there is some overlap between the individual gases and the ME mixture; as shown in the top left corner, the ethanol (E) response and the ME response overlap somewhat. This overlap could be eliminated if different factors were plotted (Factor 1 vs Factor 3, for example), but at the expense of different analytes and mixtures 
overlapping elsewhere. To obtain even better separation, one might consider using a different combination of sensing materials (more selective to either methanol or ethanol); this will be discussed further in what follows. However, the fact that methanol and ethanol responses fall in the same quadrant does make physico-chemical sense. Since the two analytes are chemically similar, it is likely that they will have a similar 'footprint' for this type of analysis.

While the plot in Figure 7 contains a very large amount of information about individual gases and related gas mixtures, the clusters for each gas combination do not show sufficient separation to use the data as a reference plot for a sensor array for these four gas analytes. To 'unclutter' the sensor array plot, new plots can be constructed using the same three sensing materials, but with combinations of up to three gas analytes (Figures 8 through 11). That is, one gas analyte and all related mixtures are excluded from each plot. Benzene is excluded in Figure 8, methanol is excluded in Figure 9, ethanol is excluded in Figure 10, and acetone is excluded in Figure 11. By dropping the number of gas analytes from four to three, the potential combinations drop from fifteen to seven. This results in significantly better separation, especially for the individual gas responses; single gas clusters are almost always independent, with the exception of benzene (which is somewhat overlapping with the MAB three-gas mixture in Figure 10).

This 'simplification' of the data also highlights the relationship between single gases, two-gas mixtures and three-gas mixtures. Take, for example, Figure 8: the three individual gases (M, E and A) are on the periphery (as we saw in Figure 7), and the two-gas mixture responses fall in between the two related single-gas responses. Similarly, the three-gas mixture response falls in the middle of the 'triangle' that forms between the three single-gas responses. 


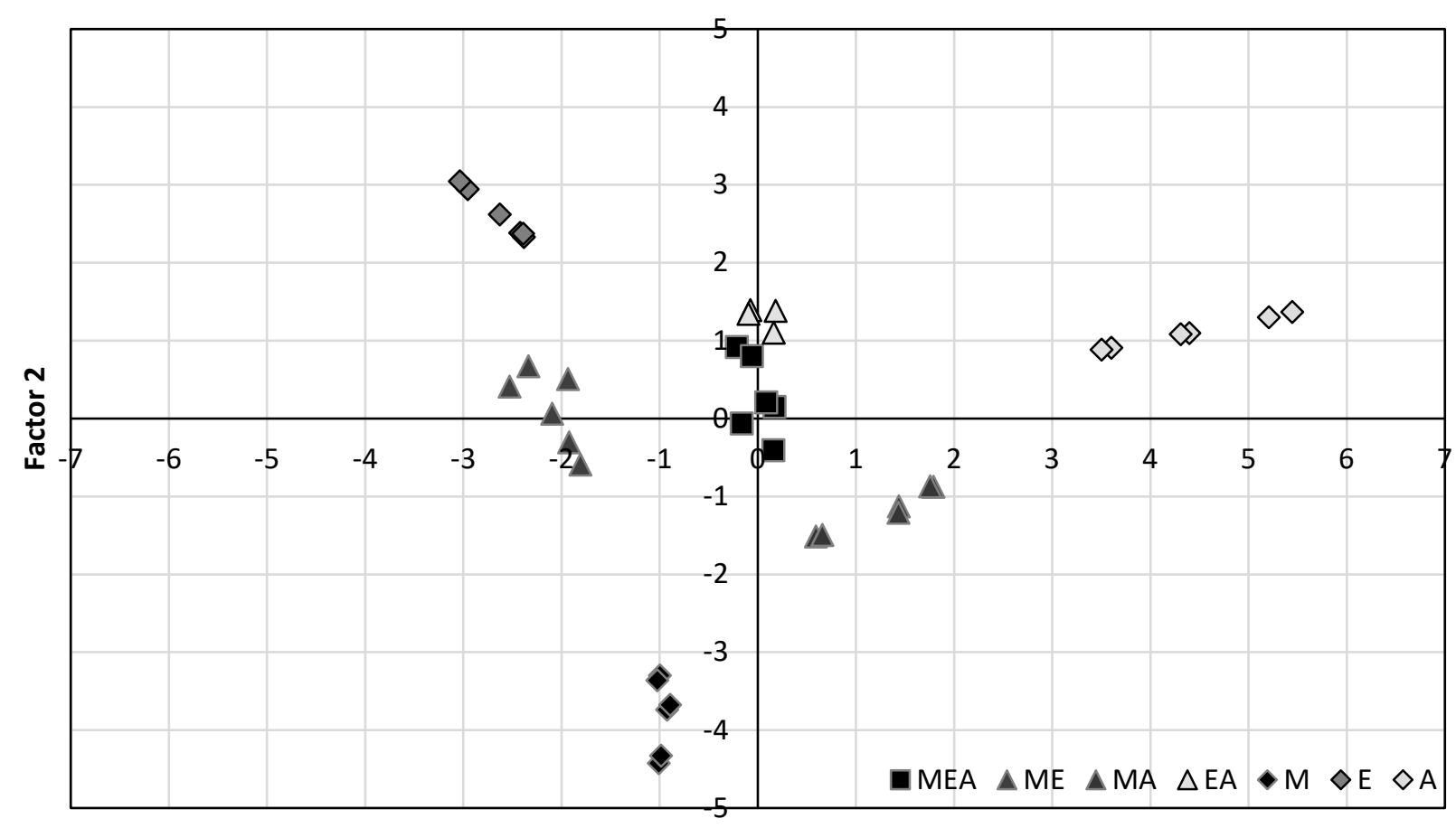

Factor 1

Figure 8 Sensor array results for all combinations of methanol (M), ethanol (E), and acetone (A) gas mixtures.

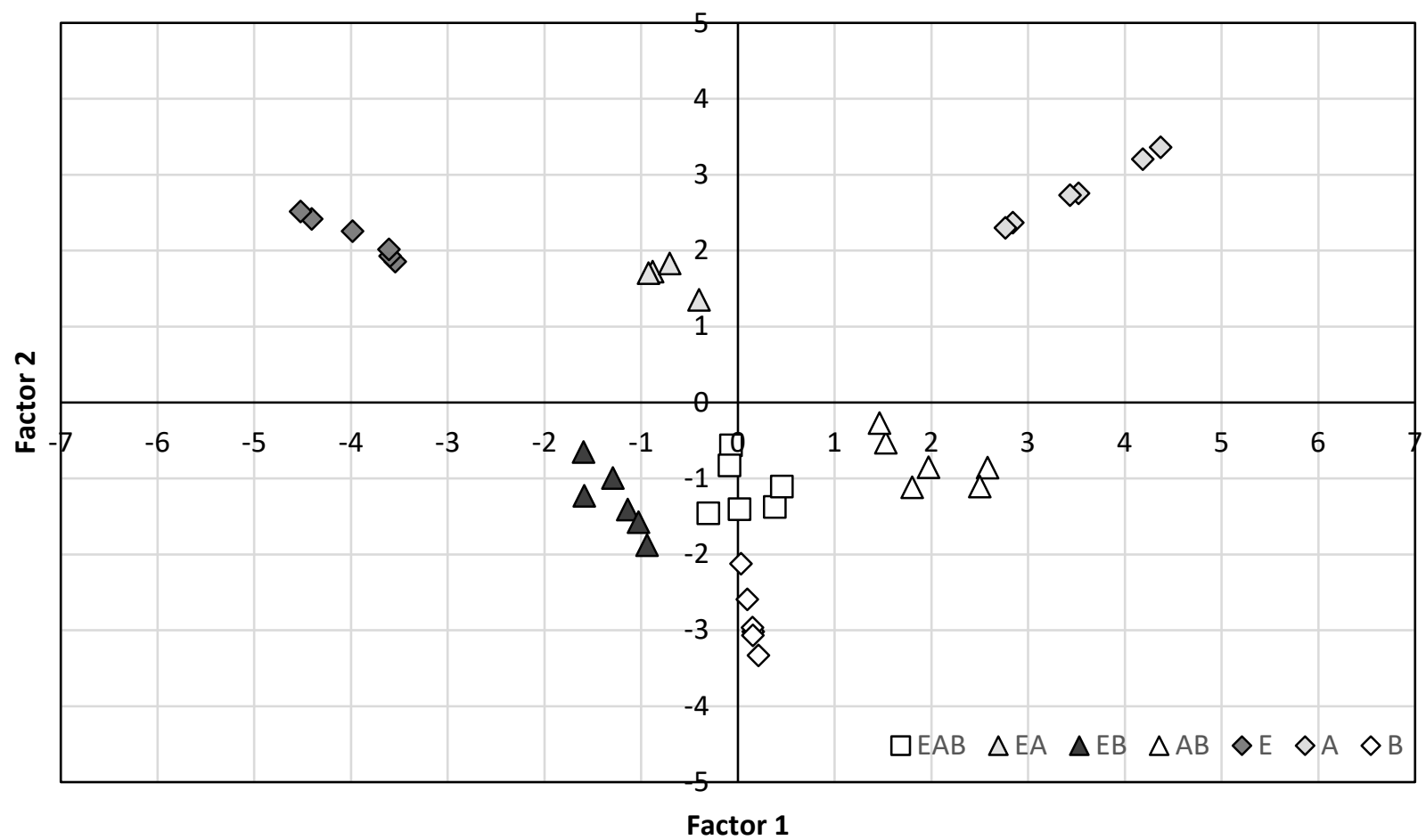

Figure 9 Sensor array results for all combinations of ethanol (E), acetone (A), and benzene (B) gas mixtures. 


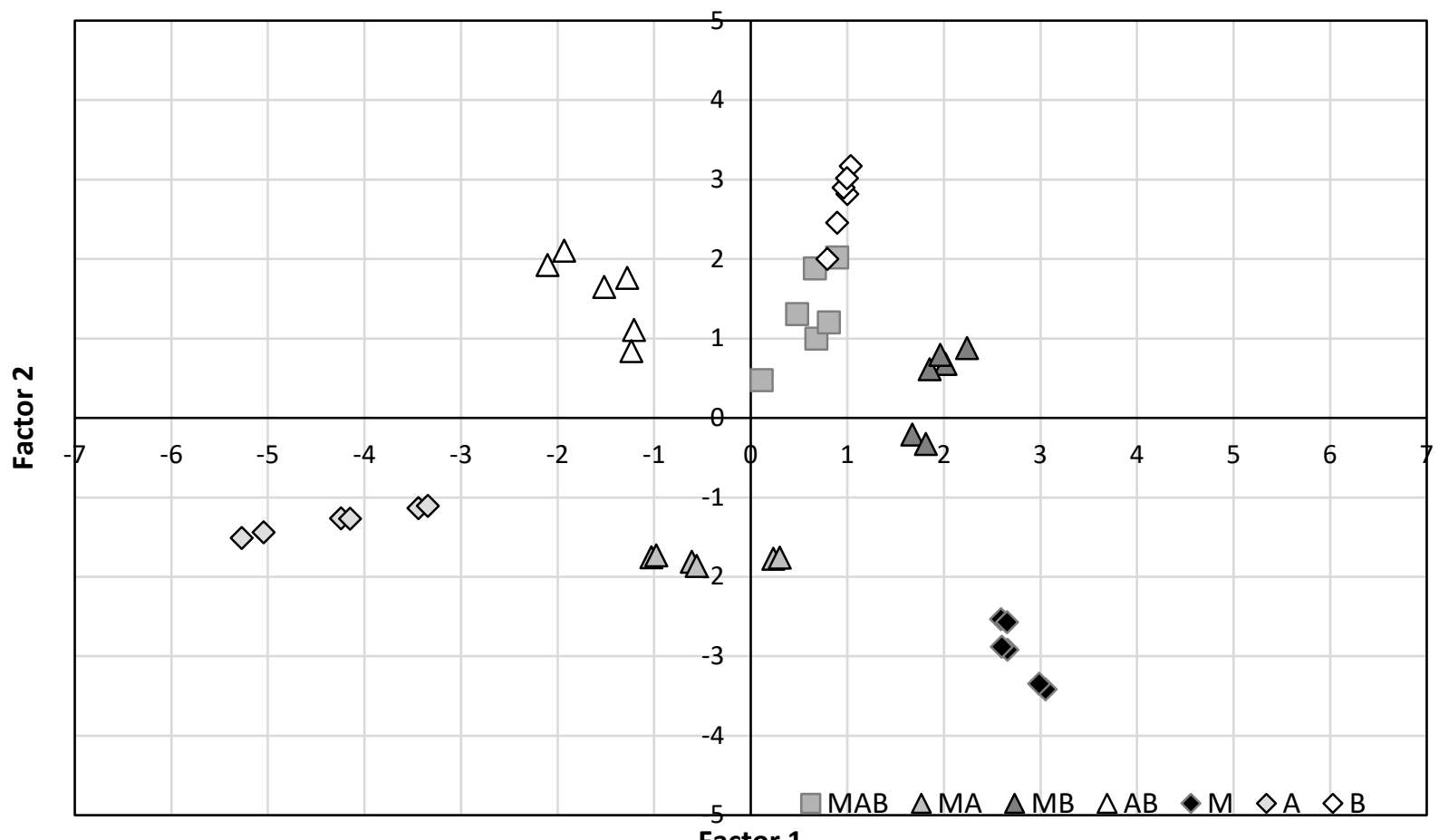

Figure 10 Sensor array results for all combinations of methanol (M), acetone (A), and benzene (B) gas mixtures.

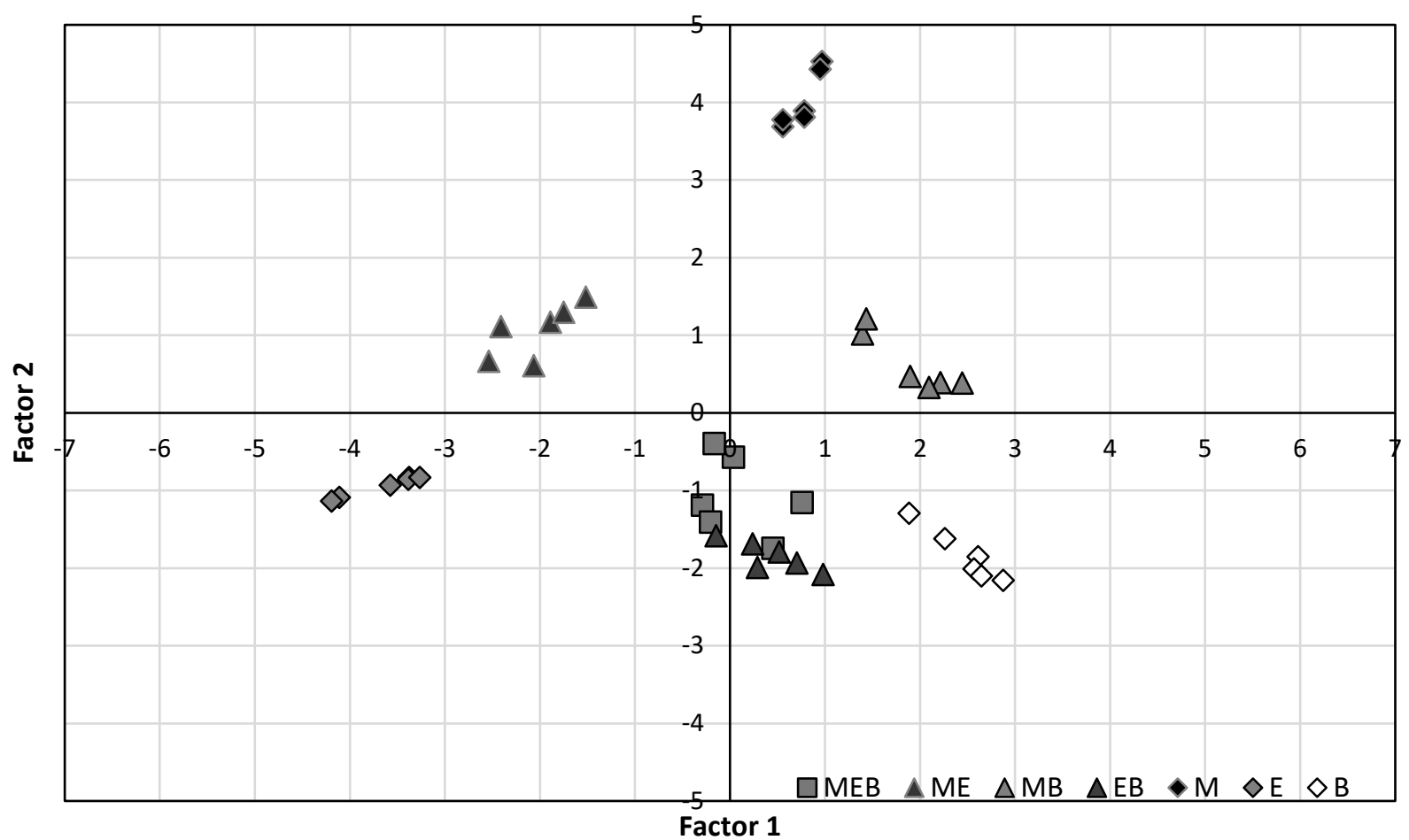

Figure 11 Sensor array results for all combinations of methanol (M), ethanol (E), and benzene (B) gas mixtures. 
Although these three-gas analyses significantly improve the separation of analytes, some overlapping responses are still observed in Figure 10 and Figure 11. In Figure 10, B and MAB responses overlap in the upper right quadrant of the plot. Similarly, in Figure 11, EB and MEB responses overlap in the lower middle portion of the plot. Interestingly, in both cases, the common analyte is benzene. The poor separation observed here may be due to the low sorption of benzene; low sorption (or low response) from experimental data limits the potential for separation of clusters using an algorithm like PCA. Also, all three PoANI-based sensing materials showed a (similar) poor affinity toward benzene (both alone and in analyte mixtures). Therefore, to improve separation in this case, one might consider using a material that has a higher affinity to benzene as part of the sensing material array.

The overlap shown on PCA plots with gas mixture data (especially Figures 7, 10 and 11) may be due to similar responses of the sensing materials to the different analytes in mixtures, the use of PCA as a clustering network, or a combination thereof. Figures 4 and 5 show similar patterns of response for the sensing materials to the combinations of gas analytes. This is especially true for PoANI and PoANI 20\% NiO. Therefore, to improve the usefulness of a sensor array, these PoANIbased sensing materials could be paired with different sensing materials (that is, different polymer backbones). In addition, a sensor array with more sensing materials could have better separation of analyte responses due to more information being included in the pattern recognition of the employed algorithm. ${ }^{23}$

For example, such a sensor array could be one consisting of PoANI, poly (vinyl pyrrolidone) $(\mathrm{PVP})^{20}$, poly (2,5-dimethyl aniline) (P25DMA) ${ }^{24}$, and SXFA ${ }^{25}$. These potential sensing materials were chosen because each one has acceptable partial selectivity to one of the four gas analytes evaluated in this paper; PoANI to acetone, PVP to ethanol, P25DMA to methanol, and SXFA to benzene. This combination of potential sensing materials may resolve the issues by having a wider variation in selectivity (to different gas analytes), as well as a larger number of sensing materials for the sensor array, which would provide more data for the pattern recognition algorithm.

Another way to identify the four gases using these three sensing materials would be to have a twostage algorithm. Stage 1 would use the PCA algorithm to plot the unknown sample against each of the three-gas mixtures (Figures 8-11). Stage 2 would compile the location on each of these four plots to determine the composition of the unknown gas sample. Although this method could work, a superior algorithm that is capable of separating these data points ${ }^{26}$ and/or a different combination of sensing materials would result in a better sensor array for these four gases ${ }^{23}$.

PCA is often used as a proof-of-concept tool because of its availability in many statistical analysis packages and its ability to group data. PCA works best when dealing with very large sets of data (thousands of data points), which means that using it as a tool to reveal the data correlation structure requires very large sets of data to create plots with which to identify unknown samples. This requires significant investment in both time and cost, as this depends on the nature of the specific experimental trials. In addition, PCA can have difficulty with noisy or drifting data, which can often occur as a sensor ages. ${ }^{27}$ 
Therefore, to improve the sensor array, these sensing materials should be combined with other sensing materials that have different response patterns to the target analytes and interferents. In addition, a better (more sophisticated) neural network or clustering algorithm could be used to better identify the gas analytes present in the sample. While this particular combination of sensing materials did not provide an ideal sensor array for methanol, ethanol, acetone, and benzene, three highly sensitive sensing materials were identified for these four gas analytes that could be used in a room temperature sensor array with other sensing materials.

Overall, these three sensing materials in combination with PCA were not ideal for creating a sensor array capable of analyzing all possible combinations of methanol, ethanol, acetone, and benzene. However, sensor arrays that were able to distinguish between three of the four analytes were developed. Since the PoANI-based sensing materials showed affinity to these four gas analytes, there is potential to use them in sensor arrays, especially for a room temperature sensor. To improve these sensor arrays, a different combination of sensing materials could be used (including some of the PoANI-based sensing materials) and/or an improved pattern recognition technique could be employed to improve the separation of the response clusters, which would allow for better identification of the gas analytes.

\section{Conclusions}

The three sensing materials (PoANI, PoANI doped with NiO, and PoANI doped with ZnO) evaluated herein had high sensitivity (showing an affinity towards the target analytes at low concentrations); however, in general, selectivity was low (that is, the sorption amount measured was similar for several distinct analytes). To improve the selectivity, the responses of these sensing materials were combined in a sensor array. A sensor array containing PoANI, PoANI 20\% NiO, and PoANI 20\% ZnO, which used PCA in a sense as an artificial intelligence algorithm, could identify the four gases (methanol, ethanol, acetone, and benzene) if individually present; however, if all four gases were present, it could not decipher all gas analyte combinations. Therefore, to create an improved sensor array, these sensing materials could be combined with other sensing materials and/or a better algorithm to fully separate different gas analytes as well as gas mixtures.

\section{Acknowledgements}

The authors are grateful for financial support from the Natural Sciences and Engineering Research Council (NSERC) of Canada and the Canada Research Chair (CRC) program.

\section{References}

1. Haugen JE, Rudi K, Langsrud S, Bredhol S. Application of Gas-sensor Array Technology for Detection and Monitoring of Growth of Spoilage Bacteria in Milk: A Model Study Anal Chim Acta. 2006;565:10-16. 
2. Di Natale C, Macagnano A, Davide F, D'Amico A, Paolesse R, Boshi T, Faccio M, Ferri G. An Electronic Nose for Food Analysis Sens Act B. 1997;44:521-526.

3. Acuña-Avila PE, Calavia R, Vigueras-Santiago E, Llobet E. Identification of Tequila with an Array of ZnO Thin Films: A Simple and Cost-Effective Method Sensors 2017;17:2943.

4. Li H, Zhang B, Hu W, Liu Y, Dong C, Chen Q. Monitoring black tea fermentation using a colorimetric sensor array-based artificial olfaction system J. Food Process Pres. 2018;42:13348.

5. Guz L, Łagód G, Jaromin-Gleń K, Suchorab Z, Sobczuk H, Bieganowski A. Application of Gas Sensor Arrays in Assessment of Wastewater Purification Effects Sensors 2015;15:1-21.

6. D'Amico A, Bono R, PennazzaG, Santonico M, Mantini G, Bernabei M, Zarlenga M, Roscioni C, Martinelli E, Paolesse R, Di Natale C. Identification of melanoma with a gas sensor array Skin Res Technol. 2008;14:226-236.

7. Turner APF, Magan N. Electronic Noses and Disease Diagnostics Nat Rev Microbiology 2004;2(2):161-166.

8. Feng L, Musto CJ, Kemling JW, Lim SH, Zhong W, Suslick KS. Colorimetric Sensor Array for Determination and Identification of Toxic Industrial Chemicals Anal Chem. 2010;82(22):9433-9440.

9. Deng C, Lv K, Shi D, Yang B, Yu S, He Z, Yan J. Enhancing the Discrimination Ability of a Gas Sensor Array Based on a Novel Feature Selection and Fusion Framework Sensors 2018;18:1909.

10. Stewart KME, Penlidis A. Sensor Array for Volatile Organic Compounds Based on Doped Poly (2,5-dimethyl aniline) Macromol Symp. 2016;370:120-127.

11. Lu Y, Partridge C, Meyyappan M, Li J. A Carbon Nanotube Sensor Array for Sensitive Gas Discrimination using Principal Component Analysis J Electrochem Chem. 2006;593:105-110.

12. Sun P, Ou Z, Feng X. Combustible Gas Discrimination by Pattern Recognition Analysis of Responses from Semiconductor Gas Sensor Array Appl Mech Mater. 2013;303-306:876-879.

13. Potyrailo RA. Polymeric Sensor Materials: Toward an Alliance of Combinatorial and Rational Design Tools? Angew Chem. 2006;45:702-723.

14. Athawale AA, Kulkarni MV. Polyaniline and its Substituted Derivatives as Sensor for Aliphatic Alcohols Sens Act B. 2000;67(1-2):173-177.

15. Stewart KME, Penlidis A. Designing Polymeric Sensing Materials for Analyte Detection and Related Mechanisms Macromol Symp.2016;360:123-132.

16. Itoh T, Matsubara I, Shin W, Izu N, Nishibori M. Preparation of Layered Organic-Inorganic Nanohybrid Thin Films of Molybdenum Trioxide with Polyaniline Derivatives for Aldehyde Gases Sensors of Several Tens ppb Level Sens Act B. 2008;128(2):512-520.

17. Na CW, Woo H-S, Lee J-H. Design of Highly Sensitive Volatile Organic Compound Sensors by Controlling NiO Loading on ZnO Nanowire Networks RSC Adv. 2012;2:414-417.

18. Stewart KME, McManus NT, Abdel-Rahman E, Penlidis A. Doped Polyaniline for the Detection of Formaldehyde J Macromol Sci A. 2012;49:1-6.

19. Stewart KME, Penlidis A. Novel Test System for Gas Sensing Materials and Sensors Macromol Symp. 2013;324:11-18.

20. Stewart KME, Penlidis A. Designing Polymeric Sensing Materials: What are we doing Wrong? Polym Advan Technol. 2017;28:319-344. 
21. Peveler WJ, Yazdani M, Rotello VM. Selectivity and Specificity: Pros and Cons in Sensing ACS Sensors 2016;1:1282-1285.

22. Jackson JEA. A User's Guide to Principal Component Analysis. New York: Wiley; 1991.

23. Gardner JW, Boilot P, Hines EL. Enhancing Electronic Nose Performance by Sensor Selection using a New Integer-based Genetic Algorithm Approach Sens Act B. 2005;106:114-121.

24. Stewart KME, Penlidis A. Evaluation of Polymeric Nanocomposites for the Detection of Toxic Gas Analytes J Macromol Sci A. 2016;53(10):610-618.

25. Chen WT, Stewart KME, Mansour RR, Penlidis A. Novel Underloaded Radio-frequency (RF) Resonant Sensor for Gaseous Ethanol and Interferents Detection Sens Act A. 2015;230:63-73.

26. Borthakur A, Cleland TA. A Neuromorphic Transfer Learning Algorithm for Orthogonalizing Highly Overlapping Sensor Array Responses. International Symposium on Olfaction and Electronic Nose; 2017 May 28-31; Montreal (QC).

27. Gardner JW, Bartlett PN. Pattern Recognition in Odour Sensing. In: Sensors and Sensory Systems for an Electronic Nose. Kluwer Academic Publishers, 1992:161-179. 